\title{
The TGF- $\beta$-Th2 axis: a new target for cancer therapy?
}

\author{
Cira García de Durango ${ }^{1}$, Maria Escribese ${ }^{2}$, and Domenico Rosace ${ }^{3}$ \\ ${ }^{1}$ DKTK Research Group \\ ${ }^{2}$ Universidad CEU San Pablo \\ ${ }^{3} \mathrm{IBMCC}$
}

March 29, 2021

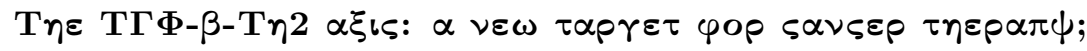

García de Durango $\mathrm{C}^{1}$, Escribese $\mathrm{MM}^{2}$, Rosace $\mathrm{D}^{3}$.

1: DKTK Research Group, Oncogenic Signalling Pathways of Colorectal Cancer, Institute of Pathology, Ludwig-Maximilians-Universität München, Munich, Germany.

2: Institute of Applied Molecular Medicine (IMMA), Department of Basic Medical Sciences, Facultad de Medicina, Universidad San Pablo-CEU, CEU Universities, Urbanización Montepríncipe, 28660 Boadilla del Monte, Madrid, Spain.

3: Centro De Investigación Del Cáncer and Instituto De Biología Molecular Y Celular Del Cáncer, Consejo Superior De Investigaciones Científicas (CSIC) - Universidad de Salamanca, Salamanca, Spain.

\section{Correspondence :}

Domenico Rosace, Centro De Investigación Del Cáncer and Instituto De Biología Molecular Y Celular Del Cáncer, Consejo Superior De Investigaciones Científicas (CSIC) - University of Salamanca, Salamanca, Spain. domenico.rosace@usal.es

\section{Funding information}

DR is supported by Juan de la Cierva Formación grant (FJC2018-038723-I) from the Spanish Ministry of Universities.

Keywords : type 2 immunity; cancer; hypoxia; IL-4; TGF- $\beta$

Abbreviations: CAR (chimeric antigen receptor), CTL-4 (cytotoxic T lymphocyte-associated protein 4), EMT (epithelial-to-mesenchymal transition), IL (interleukin), IT (immunotherapy), PD-1 (programmed cell death protein 1), PD-L1 (programmed cell death ligand 1), Th (T helper cell), TGF- $\beta$ (transforming growth factor $\beta$ ), TGFR2 (TGF- $\beta$ receptor 2$)$.

\section{MANUSCRIPT}

The innate and adaptive immune responses are critical for the recognition and removal of pathogens. Moreover, in the last two decades it has been demonstrated that immune cells are also key cells in the cancer-related immune response. ${ }^{1}$ Ideally, the immune system should eradicate the tumor cells to maintain homeostasis. However, it has been demonstrated that a dysregulation of the innate and adaptive immune responses could lead to tumorigenesis, inhibition of $\mathrm{T}$ - and B-cell activation, and stimulation of tumor proliferation and metastasis. $^{2}$ The immune system can also promote tumor progression through a dynamic process called cancer immunoediting, by which cancer cells acquire mutations that allow them to evade the immune system. Tumor cells that undergo this process harbour a reduced immunogenicity and produce regulatory 
cytokines such as interleukin-10 (IL-10) and transforming growth factor $\beta$ (TGF- $\beta$ ) that can inhibit T-cell functionality. ${ }^{2}$

The role of T cells in tumor immunity has been extensively studied. Several subsets of CD4+ T helper (Th) cells are involved in tumor immunity, but the main ones are Th1 and Th2. Each type produces a different set of cytokines and present divergent outcomes in cancer immunity. Th1 responses are frequently described as antitumoral because they contribute to the activation of cytotoxic CD8 T cells and macrophages, which has been linked to tumor regression. However, the role of Th2 immunity in cancer is controversial. Th2 cells produce pro-inflammatory cytokines such as IL-4, IL-5 and IL-13, leading to inflammation, which can promote tumor progression. On the other hand, the same Th2-related cytokines can exhibit antitumoral activity through eosinophil recruitment in the tumor environment. ${ }^{3}$ Th1 and Th2 immunity can be regulated by multiple mechanisms. As aforementioned, the pleitropic cytokine TGF- $\beta$ is able to inhibit immune responses by blocking Th1 and Th2 effector cell activity. In the tumor environment, the re-activation of cytotoxic T-cell responses via TGF- $\beta$ inhibition has been proposed as an approach for cancer immunotherapy (IT).

Cancer IT, described as a type of therapy that reduces tumor burden by inducing or suppressing the immune system, has become a pivotal strategy for cancer IT. It usually follows two different strategies. The first is the inhibition of immune checkpoints responsible to induce tolerance, which empowers the natural function of the immune system. In this context, the inhibitors targeting cytotoxic $\mathrm{T}$ lymphocyte-associated protein 4 (CTLA-4) and programmed cell death protein 1/programmed cell death ligand 1 (PD-1/PD-L1) have emerged as the gold standard of care regimens for patients with different cancer types. ${ }^{4}$ The second approach involves T-cell engineering to express chimeric antigen receptors (CAR-T) that specifically detect the cancer cell. Considering their use and therapy in a broad spectrum of cancer types, they constitute a potent new cancer therapy. ${ }^{5}$

The recent article by Liu et al . investigated type 2 immune responses and a cancer immune tolerance mechanism mediated by TGF- $\beta .^{6}$ They used a mouse model of metastatic breast cancer and depleted TGF- $\beta$ receptor 2 (TGFR2) in lymphocytes. When TGF- $\beta$ signalling was inhibited in CD4+ lymphocytes, the tumor tissue underwent remodelling: the vasculature became less leaky and large tumor regions remained avascular, thus creating a hypoxic region where cells undergo apoptosis. These changes appeared to be mediated through the hallmark Th2 cytokine IL-4, and the authors showed an IL-4-dependent gene signature that correlates to survival in other cancer types (Figure 1 ). ${ }^{7}$

In this regard, the IL-4 axis is drawing an increasing interest as a target for pathologies with an immune underlying mechanism. The blockade of the IL- $4 \mathrm{R} \alpha$ (the receptor for of IL- 4 and IL-13) with monoclonal antibodies such as dupilumab has emerged as a promising strategy for the treatment of certain allergic diseases such as atopic dermatitis and allergic asthma. ${ }^{8}$ Given the findings from Liu et al ., where IL-4 acts as the downstream mediator of TGF- $\beta$ pro-tumor effects, cancer patients may benefit from IL-4R $\alpha$ blockade.

Liu et al . found IL-4 effects to be mediated through blood vessel remodelling: when TGF- $\beta$ siglaning is specifically blocked in CD4+ lymphocytes, it occurs a rearrangement of the vasculature that leaves large tumor areas without irrigation. Consequently, they experience hypoxia and prone to cell death.

Regardless of the potential of the discovery, hypoxia signalling has been studied to influence the first steps of the metastatic process. Hypoxia has been shown to promote Epithelial-to-mesenchymal transition (EMT) ${ }^{6}$ but also to mediate resistance mechanisms in anticancer therapy ${ }^{9}$ and to shape certain circulating tumor cells to become highly metastatic. ${ }^{10}$ Ye and collaborators ${ }^{9}$ established a panel of genes to monitor the effects of tumor hypoxia on treatment sensitivity and resistance, including IT. On this subject, a personalized approach may be needed.

The discovery that TGFR2 deletion in CD4+ cells leads to tumor cell death in a metastatic breast cancer animal model through vasculature remodelling opens new interesting therapeutic lines. Nonetheless, the possible resistance mechanisms due to the ambiguity of hypoxia effects remain to be elucidated. 
Altogether, these discoveries demonstrate the importance of personalized medicine for the design of potential therapeutic strategies in cancer. Novel IT strategies including hypoxia-mediated therapy response-sensitivity may potentiate CD4+ T-cell effects by altering the TGF- $\beta$ pathway, thus improving cancer patients prognosis.

Figure 1 : In a breast cancer animal model, Liu et al found that TGF- $\beta$ signalling on CD4+ cells contributed to tumor growth and neoangiogenesis. When the signalling was turned off specifically in Th2 cells, large tumor areas remained hypoxic due to the lack of vasculature, and prone to undergo cell death. These effects were mediated through effector cytokine IL-4.

\section{ACKNOWLEDGMENTS}

The authors recognize Dr. Anna Globinska for graphical abstract design and Dr. Rodrigo Jiménez-Saiz for critical review of the manuscript.

\section{CONFLICTS OF INTEREST}

The authors have no conflict of interest to declare.

\section{AUTHOR CONTRIBUTION}

The authors approved the final version of the manuscript as submitted and agreed to be accountable for all aspects of the work.

\section{ORCID}

Cira García de Durango: 0000-0002-1076-0148

María Marta Escribese Alonso: 0000-0001-5057-5150

Domenico Rosace: 0000-0002-8833-8422

\section{REFERENCES}

1. Finn OJ. Immuno-oncology: understanding the function and dysfunction of the immune system in cancer. Ann Oncol Off J Eur Soc Med Oncol2012;23 Suppl 8 :viii6-9.

2. Cervantes-Villagrana RD, Albores-García D, Cervantes-Villagrana AR, García-Acevez SJ. Tumor-induced neurogenesis and immune evasion as targets of innovative anti-cancer therapies. Signal Transduct Target Ther $2020 ; 5: 99$.

3. Carretero R, Sektioglu IM, Garbi N, Salgado OC, Beckhove P, Hämmerling GJ. Eosinophils orchestrate cancer rejection by normalizing tumor vessels and enhancing infiltration of CD8(+) T cells. Nat Immunol 2015;16:609-617.

4. Ellis GI, Riley JL. How to kill Treg cells for immunotherapy.Nat Cancer 2020;1 :1134-1135.

5. Labanieh L, Majzner RG, Mackall CL. Programming CAR-T cells to kill cancer. Nat Biomed Eng 2018;2 :377-391.

6. De Bock K, Mazzone M, Carmeliet P. Antiangiogenic therapy, hypoxia, and metastasis: risky liaisons, or not? Nat Rev Clin Oncol2011;8 :393-404.

7. Liu M, Kuo F, Capistrano KJ, Kang D, Nixon BG, Shi W et al. TGF- $\beta$ suppresses type 2 immunity to cancer. Nature 2020;587:115-120.

8. Gandhi NA, Bennett BL, Graham NMH, Pirozzi G, Stahl N, Yancopoulos GD. Targeting key proximal drivers of type 2 inflammation in disease. Nat Rev Drug Discov 2016;15 :35-50.

9. Ye Y, Hu Q, Chen H, Liang K, Yuan Y, Xiang Y et al. Characterization of Hypoxia-associated Molecular Features to Aid Hypoxia-Targeted Therapy. Nat Metab 2019;1 :431-444. 
10. Donato C, Kunz L, Castro-Giner F, Paasinen-Sohns A, Strittmatter K, Szczerba BM et al. Hypoxia Triggers the Intravasation of Clustered Circulating Tumor Cells. Cell Rep 2020;32:108105.

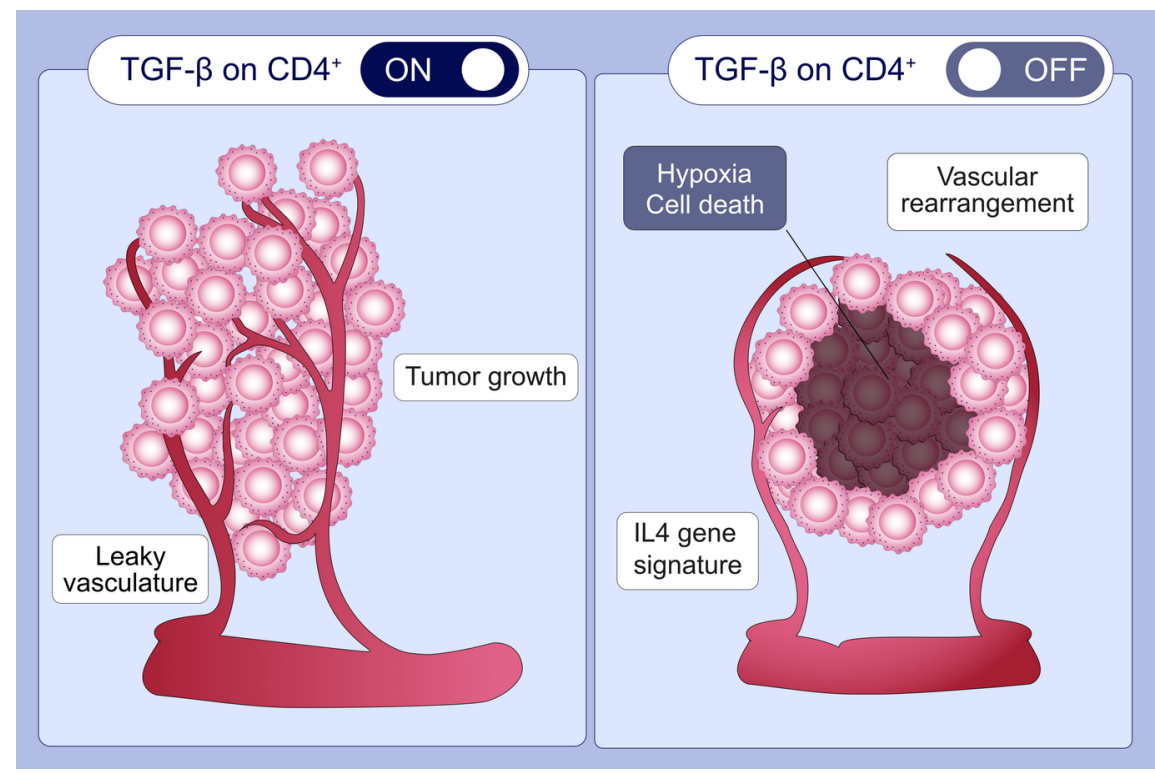

